\title{
Hemispheric Asymmetry for Language Processing and Lateral Preference in Simultaneous Interpreters
}

\author{
Alice Mado Proverbio, Roberta Adorni \\ Lab of Cognitive Electrophysiology, Department of Psychology, \\ University of Milano-Bicocca, Milan, Italy \\ Email: mado.proverbio@unimib.it \\ Received October $5^{\text {th }}, 2010$; revised December $16^{\text {th }}, 2010$; accepted December $19^{\text {th }}, 2010$.
}

\begin{abstract}
Neuroimaging studies showed that linguistic functions are less lateralized in polyglots than in monolinguals. However, there is not much agreement about the role of the two hemispheres in semantic and syntactic processing in bilinguals. In this study, 35 right-handed Italian speakers were shown 520 words and pseudo-words. The task consisted in detecting a given target letter by pressing a button with either the left or right hand. 19 simultaneous interpreters and 16 monolingual University students participated in the study. Interpreters performed the task in their native (L1) and second language (L2 = English); monolingual students only in L1. Response times to targets were recorded as a function of the hand used. RTs were faster to words than pseudo-words (word superiority effect). Results showed a significant right hand/LH advantage for the student group, and a complete lack of asymmetry for the interpreters both in L1 and L2. These data indicate a left-lateralization of linguistic functions in monolinguals and reduced lateralization in polyglots. The lack of lateralization in interpreters can be attributed either to their polyglottism, or to their prolonged practice of simultaneous interpreting strategies (e.g., dealing with two input channels; right ear/LH for listening to themselves interpret and left ear/RH for listening to the source language).
\end{abstract}

Keywords: Laterality, Hemispheric Asymmetry, Interpreters, Bilingualism, Brain

\section{Introduction}

It is known that linguistic functions are left lateralized in the human brain, and that about $85 \%$ of the world's population is right-handed. While the great majority of right-handers show a left lateralization for language, about $25 \%$ of left-handers might present a certain right lateralization of some linguistic functions (Hugdahl \& Davidson, 2003). Reduced lateralization might be linked to faster inter-hemispheric transfer times, to a thicker corpus-callosum, or to some functional specialization. The cerebral organization of linguistic functions in bilinguals also depends on variation in language experience such as the age of acquisition of both foreign and native languages, the degree of proficiency, the context of acquisition (affective vs. scholar), the exposure to different linguistic environments. One way to investigate the brain lateralization of linguistic functions controlling for the level of proficiency is to study simultaneous interpreters. They master multiple languages to the highest proficiency levels because of their specialized studies and their professional activity. The observation of these individuals offers the possibility to control for the age of acquisition of native vs. foreign languages, and to establish the exposure to specific linguistic environments. Unfortunately, to our knowledge, very few cognitive studies on lateral preference and multilingualism have been performed on simultaneous interpreters, and even fewer of them have performed direct brain measurements.

A number of behavioural studies were performed with interpreters in the nineties. For example, Lambert (Lambert, 1989) showed that interpreters performed significantly better interpreting from the non-native language or second language (L2) to the mother tongue or first language (L1) when the message in L2 was sent to the left ear (right hemisphere). She suggested that in this condition, the right ear (left hemisphere) was prevailingly involved in the interpretation process and in the monitoring of the outgoing speech, while the left ear (right hemisphere) was more specialized in the reception of the incoming information. This study validated the observation of cerebral lateralization in proficient bilinguals and, at the same time, also revealed the symmetrical cerebral involvement in simultaneous interpretation. In another study Green et al. (Green et al., 1990) observed a significantly higher involvement of the left hemisphere during a shadowing task, which requires participants to repeat spoken words or sentences out loud, and no asymmetry during an interpreting task. This study supported the hypothesis that simultaneous interpretation involves both cerebral hemispheres, which Green et al. (Green et al., 1990) attribute to the complexity of the linguistic and cognitive processes involved. In addition the authors, having performed the interpreting experiment with non-interpreter as well as interpreter bilinguals, suggested that the great symmetry found in the interpreting task in both groups was task-related and not a result of professional interpreter training.

A series of interesting experiments were carried out by Fabbro et al. (Fabbro et al., 1990) in which language processing was compared in right-handed interpreting students and monolingual medical students. No significant cerebral lateralization was found for L1 either in the interpreting students or in the control group in an automatic speech production task. However, using a verbal-manual interference paradigm a significantly higher degree of verbal-manual interference was found for L1 than for L2 and L3 in simultaneous interpreters, thus suggesting 
the involvement of less lateralized linguistic processes for languages acquired later. In a similar investigation, hemispheric specializations for semantic and syntactic components in Italian (L1) and English (L2) were studied with a dichotic listening test involving text comprehension in right-handed interpretation students and professional interpreters (Fabbro \& Gran, 1991). In regard to hemispheric specialization in interpreting students, no significant asymmetries were revealed in the recognition of semantic and syntactic errors. Interpreters showed a right-ear superiority in recognizing semantic errors in L1 and a significant left-ear superiority in recognizing semantic errors in L2. In the recognition of syntactic errors, interpreters showed significant left-ear superiority for L1 and significant right-ear superiority for L2. The authors interpreted their pattern of results as being due to the prolonged practice with simultaneous interpreting strategies as professional interpreters. This experience might have accounted for some of their peculiar hemispheric specializations for languages.

In a recent study, Proverbio et al. (Proverbio et al., 2007) compared response times to words expressed in L1 or in a perfectly mastered foreign language in individuals who had been bilingual since birth (early bilinguals), individuals who had learned a second language before puberty and worked as simultaneous interpreters (interpreters), and individuals who had never learned a second language (monolingual controls). In the first experiment, subjects judged the meaningfulness of short L1 and L2 sentences. The analysis of response speed revealed a functional hemispheric asymmetry. Indeed, when they used their left hands, the three groups did not statistically differ from each other. However, both controls and interpreters were faster than bilinguals when using their right hand. This suggests that the age of acquisition of L2 may have an effect on the functional organization of the linguistic brain. Interestingly, while both controls and interpreters were faster when using their right hand, bilinguals were faster when using their left hand, which suggests a greater involvement of the right hemisphere in linguistic processing in native fluent bilinguals (Fabbro \& Gran, 1991; Proverbio et al., 2002; Proverbio et al., 2004). In a second experiment (Proverbio et al., 2007) a group of simultaneous interpreters was asked to identify a given letter (orthographic detection task), not taking into account if the target letter was embodied in a word or pseudo-word, in their L1, L2 or L3. The results revealed no hand asymmetry. The authors concluded that the lateralization effects found in bilinguals and interpreters supported the hypothesis that the difference across the two groups could be based on the different ages of acquisition of L2 and on the relative different functional organization of the linguistic brain.

In one of the few neuroimaging studies on interpreters, Rinne et al. (Rinne et al., 2000) performed a PET study on Finnish/English interpreters during simultaneous interpreting vs. shadowing of auditorily presented text. The authors found that interpreting into L2 elicited a much more extensive left fronto-temporal activation than interpreting into L1, thus suggesting that interpreting into the non-native language was more demanding than in to L1. In a study performed by our research group (Proverbio et al., 2004) event related potentials (ERPs) were recorded in native Italian interpreters and controls during a semantic task in which the subjects judged the meaningfulness of short Italian and English sentences in L1/L2 mixed or unmixed conditions. Overall, both RTs and electrophysiological data indicated a lesser degree of hemispheric lateralization for linguistic function during L2 rather than L1 processing in interpreters. Indeed, both interpreters and controls produced faster responses with their right hand and interpreters were faster at responding to L1 words than L2 ones. Interpreters also showed a greater asymmetry between hands with L1 words, with a significant advantage for the right hand with Italian, but not English words. Evoked potential responses suggested less hemispheric asymmetry in N1 component distribution for L2 sentences than for L1 ones. In another ERP study, Proverbio et al. (Proverbio et al., 2009) investigated the timing of brain activation during the processing of L1 vs. languages mastered equally (L2) or less (L3) proficiently than L1 in simultaneous interpreters during an orthographic task. The data showed marked differences in ERPs related to linguistic processes for native vs. foreign languages and deeply mastered vs. not mastered (L2 vs. L3) languages, tracing clear markers of age of acquisition and language proficiency. No differences in hemispheric asymmetry were found as a function of language, suggesting a reduced left lateralization of linguistic processes in simultaneous interpreters.

The aim of the present study was to shed light on this matter by investigating whether interpreters are less lateralized than non-polyglots in processing L1 words. We compared the behavioural performance of interpreters vs. monolinguals on a letter detection task. Assuming that the orthographic task requires basic linguistic processes as compared to the complexity of simultaneous interpretation, we hypothesized that symmetrical involvement of both cerebral hemispheres in the letter detection task might be attributed to less lateralization for language functions in polyglots, rather than the simultaneous interpretation task perse.

\section{Methods}

\section{Experiment 1}

\section{Participants}

Nineteen native Italian female professional interpreters took part in the experiment. They were all right-handed and aged between 28 and 53 years $($ Mean $=42 ; \mathrm{SD}=6.27)$. All had normal or corrected-to-normal vision and were in good health; none had ever suffered from neurological or psychiatric disorders. Handedness was assessed by the Italian version of the Edinburgh Inventory Questionnaire (Oldfield, 1971). Their mean lateral preference value (ranging from -1 to 1 ) was 0.91 ( $\mathrm{SD}=$ 0.14 ). They were all graduates in Languages and Foreign Literature and specialized in conference interpreting and practiced simultaneous English $<>$ Italian $(\mathrm{EN}<>$ IT) interpreting. Experiments were conducted in accordance with ethical standards (Helsinki, 1964).

\section{Stimuli and Procedure}

A total number of 520 stimuli were used. They consisted of words (130 Italian and 130 English words) and legal pseudowords, namely pseudo-words that were consistent with the specific orthography of each language (130 Italian and 130 English pseudo-words) and were presented one at a time at the center of a computer screen (see Table 1 for some examples of stimuli). All stimuli were typed in Times font and were white on a black background. The lengths of the stimuli ranged from 
Table 1.

Examples of words and pseudo-words of different length (6-10 letters) and language ( $L 1$ or $L 2$ ) presented to interpreters and monolingual subjects.

\begin{tabular}{|c|c|c|}
\hline \multicolumn{3}{|c|}{ Interpreters (L1) } \\
\hline$\#$ & Words & Pseudo-words \\
\hline 6 & sedano (celery) & inallo \\
\hline 7 & proroga (extension) & fersado \\
\hline 8 & briciola (crumb) & vualneto \\
\hline 9 & borghesia (gentry) & qualverio \\
\hline 10 & bomboniera (wedding keep-sake) & buncarpeve \\
\hline \multicolumn{3}{|c|}{ Interpreters (L2) } \\
\hline 6 & knight & pleath \\
\hline 7 & dreamer & skeange \\
\hline 8 & twilight & spounger \\
\hline 9 & blueberry & smitchler \\
\hline 10 & disclaimer & genbiscrow \\
\hline \multicolumn{3}{|c|}{ Monolinguals (L1) } \\
\hline 6 & ascesa (ascent) & nelebo \\
\hline 7 & agnello (lamb) & menozio \\
\hline 8 & metafora (metaphor) & feriduso \\
\hline 9 & trattoria (restaurant) & meascetto \\
\hline 10 & migrazione (migration) & gristultro \\
\hline
\end{tabular}

4 to $7 \mathrm{~cm}$. They were $1 \mathrm{~cm}$ in height and subtended visual angles of $0^{\circ} 25^{\prime} 48^{\prime \prime}$ in the vertical axis and $1^{\circ} 43^{\prime} 12^{\prime \prime}$ to $3^{\circ} 1$ ' $12^{\prime \prime}$ in the horizontal axis.

The stimuli were presented in blocks of 13 trials each of which lasted about $1.5 \mathrm{~min}$ and was preceded by 3 warning signals "ready", “set', “go" presented for $250 \mathrm{~ms}$. Each stimulus was presented individually and remained on the screen for $200 \mathrm{~ms}$ and was followed by a $1400-1600 \mathrm{~ms}$ random inter-stimulus interval (ISI). Participants were instructed to look at a fixation point located in the center of the screen. Thirteen different characters were used as target letters through the different blocks. At the beginning of each run, participants were informed of the target letter for the following run. They were then asked to respond as accurately and quickly as possible to the presence of target letters by pressing a response key with the index finger of the left or right hand. Participants were asked to take no account of the language (Italian or English) or of whether the word was congruous or non-existent. Both hands were used alternately during the recording session. The order of hand use and the task conditions were counterbalanced across subjects. For each sequence, half of the stimuli were targets and the other half were non-targets. All stimuli were balanced in terms of length and position of target letter (beginning, middle or end of word). Words were balanced in terms of imageability and frequency.

\section{Statistical Analysis}

For each participant, reaction times longer than $1000 \mathrm{~ms}$ or exceeding of mean \pm 2 standard deviations were excluded. Mean reaction times were subjected to a multifactorial repeated-measure ANOVA. Factors included language (Italian,
English), response hand (right, left) and word type (word, pseudo-word).

\section{Results}

An ANOVA performed on RTs indicated a significant effect of language $(\mathrm{F}(1,18)=32.594 ; \mathrm{p}<0.001)$. The interpreters were faster in responding to Italian stimuli than to English stimuli $($ Italian $=562 \mathrm{~ms}, \mathrm{SD}=46.96$; English $=585 \mathrm{~ms}, \mathrm{SD}=$ 43.34). The effect of word type $(F(1,18)=33.574 ; p<0.001)$ showed that participants were faster in responding to words than to pseudo-words (Words $=564 \mathrm{~ms}, \mathrm{SD}=43.08$; Pseudowords $=584 \mathrm{~ms}, \mathrm{SD}=47.78)$. No effect of hand was observed, which means that for both languages (Italian and English) interpreters showed a bilateral hand use (Italian: $\mathrm{RH}=564 \mathrm{~ms}$, $\mathrm{LH}=560 \mathrm{~ms}$; English: $\mathrm{RH}=581 \mathrm{~ms}, \mathrm{LH}=589 \mathrm{~ms}$ ), as displayed in Figure 1 (left).

\section{Experiment 2}

\section{Participants}

Sixteen university students ( 7 men, 9 women) took part in this experiment. Participants were native Italian and had a scholastic knowledge of foreign languages. They were all right-handed and aged between 20 and 29 years $($ Mean = 23; $\mathrm{SD}=2.26$ ). All had normal or corrected-to-normal vision and were in good health; none had ever suffered from neurological or psychiatric disorders. As in the previous study, handedness was assessed by the Italian version of the Edinburgh Inventory Questionnaire. Their mean lateral preference value was 0.86 $(\mathrm{SD}=0.15)$. There was no significant difference between interpreters and monolinguals in terms of preference value $(\mathrm{t}=$ $1.20 ; \mathrm{p}=0.24)$. As with experiment 1 , this experiment was conducted in accordance with ethical standards (Helsinki, 1964).

\section{Stimuli and Procedure}

Stimuli consisted of 300 words and 300 legal pseudo-words (see Table 1 for some examples of stimuli). They were presented one at a time at the center of a computer screen, they were typed in Arial Narrow font, and they were white on a black background. Their length varied from 5 to $8 \mathrm{~cm}$, they

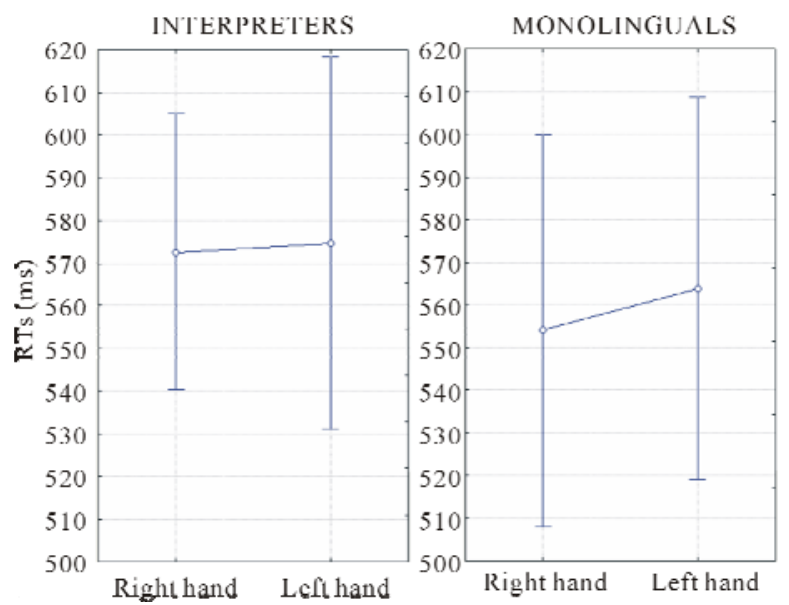

Figure 1.

Reaction times (and standard deviations) recorded in the interpreter and monolingual groups, as a function of the hand used to respond. 
were $1 \mathrm{~cm}$ in height and they subtended visual angles of $0^{\circ} 30^{\prime} 10^{\prime \prime}$ in the vertical axis and $2^{\circ} 31^{\prime} 51^{\prime \prime}$ to $4^{\circ} 1{ }^{\prime} 22^{\prime \prime}$ in the horizontal axis. Each block of trials lasted about $1.5 \mathrm{~min}$ and was preceded by 3 warning signals "ready", "set", "go", presented for $800 \mathrm{~ms}$. Each stimulus remained on the screen for $200 \mathrm{~ms}$ and was followed by a $1400-1600 \mathrm{~ms}$ random ISI. Subjects were instructed to stare at a fixed point located in the center of the screen. Ten different characters were used as target letters through the different runs. At the beginning of each run, participants were informed of the target letter for the following run. As in the previous experiment, the task consisted of responding as accurately and quickly as possible to the presence of target letters by pressing a response key with the index finger of the left or right hand, taking no account of whether the word was congruous or non-existent. The two hands were used alternately during the recording session. The order of hand use and the task conditions were counterbalanced across subjects. For each sequence, half of stimuli were targets and the other half were non-targets. All stimuli were balanced in terms length and position of target letter (beginning, middle or end of word).

\section{Statistical Analysis}

For each participant, reaction times longer than $1000 \mathrm{~ms}$ or exceeding of mean \pm 2 standard deviations were excluded. Mean reaction times were subjected to a multifactorial repeatedmeasures ANOVA. Factors included response hand (right, left) and word type (word, pseudo-word).

\section{Results}

An ANOVA performed on RTs showed a significant effect of hand $(\mathrm{F}(1,15)=5.792 ; \mathrm{p}<0.05)$, which indicated that the monolinguals were faster in responding with their right hand than left hand $(\mathrm{RH}=554, \mathrm{SD}=62.36$; $\mathrm{LH}=564, \mathrm{SD}=59.59)$. Consistent with the data from interpreters, the effect of word type $(\mathrm{F}(1,15)=27.458 ; \mathrm{p}<0.001)$ suggested that participants were faster in responding to words than to pseudo-words (Words $=549 \mathrm{~ms}, \mathrm{SD}=62.63$; Pseudo-words $=569 \mathrm{~ms}, \mathrm{SD}=$ 57.86).

\section{Discussion}

In this study the performance of a group of native Italian interpreters and monolingual University students in a letter detection task was observed. Response times to targets were recorded and compared across groups. For both groups, RTs were faster to words than pseudo-words. This well documented effect is known as the word superiority effect (Reicher, 1969). It refers to the phenomenon that people are more accurate in recognizing a letter in the context of a word than they are when a letter is presented in isolation, or within a non-word. The effect suggests that words are not processed letter-by-letter, but rather that letter recognition is inhibited or facilitated according to top-down information about the word-context. Interpreters were faster at responding to L1 words than L2 ones. This result is in agreement with the findings of previous studies of our research group (Proverbio et al., 2007; Leoni et al., 2004) and is reasonably related to the age of $\mathrm{L} 2$ acquisition.

More interestingly for the purpose of the present paper, results showed a marked right hand advantage in response speed in University students and no differences between hands in interpreters. Since stimuli were foveally presented in this study, the hand advantage for the monolingual group might be interpreted as a sign of left lateralization of the cognitive functions required by the task, namely orthographic search and analysis. Indeed neuroimaging studies support the hypothesis that the cortical area devoted to letter and word processing, known as "Visual Word Form Area" (Cohen \& Dehaene, 2004; Cohen et al., 2002; Polk et al., 2002) and corresponding to the fusiform gyrus of the temporal cortex, is strongly lateralized to the left hemisphere (Flowers et al., 2004; Proverbio et al., 2007; Zani et al., 2008; Xue et al., 2006). Although we are not aware of other evidence supporting the view that simultaneous interpreters might be less lateralized for reading, such a pattern has been shown here. There is some evidence for non-interpreters. In an electrophysiological study (Proverbio et al., 2002), Slovenian / Italian bilinguals showed a strong left-sided activation of the occipito-temporal regions dedicated to orthographic processing reflected by the N1 component of event-related potentials for L1 words, but a bilateral activation of the same areas for L2 words. In our view, this indicates that the word form system might be able to discriminate between different languages on the basis of orthographical analysis at very early stages of visual processing. In polyglots, this early detection would enable the reader to address the specific knowledge proper to a given language (i.e., graphemic/phonemic conversion rules, lexicon, etc.) in order to comprehend the material. In fact, the bilinguals from Proverbio and colleagues' study (Proverbio et al., 2002), had an involvement of the right lateral occipital area in the orthographic analysis of L2 words, as reflected by the topographical distribution of the early-latency N1 component. Overall, these data suggest a greater involvement of the right hemisphere in the linguistic processing of L2 (compared to L1) in apparently highly fluent early bilinguals, suggesting a possible preference for the mother tongue. It is very important to note that this preference did not produce overall faster RTs for $\mathrm{L} 1$ than for L2. The L1 preference was subtly revealed in the electrophysiological responses, for example, by very small or absent N400 or P600 to syntactically incongruent Italian words.

These findings are compatible with other evidence in the literature suggesting that hemispheric lateralization of linguistic functions is different in polyglots. Chernigovskaya and coauthors (Chernigovskaya et al., 1983) showed a different lateralization of semantic and syntactic structures for the first language (L1) and the second (L2) in a bilingual psychiatric patient treated with unilateralized electroconvulsive therapy. The authors described a lateralization of semantic functions to the right hemisphere for L1 and to the left hemisphere for L2, while syntactic functions were lateralized to the left hemisphere for both languages. A differential lateralization of multiple languages has also been reported for professional interpreters, as mentioned in the introduction section (Fabbro et al., 1990; Gran et al., 1991). However, the pattern of lateralization in these individuals is complicated by an asymmetric use of the ears for listening to the linguistic material to be translated during their professional commitments. Interpreters have a habit of listening to the source language with the left ear (right hemisphere) and taking off the right earphone in order to leave the right ear (left 
hemisphere) free to monitor their own verbal performance (Gran \& Fabbro, 1989).

Clinical cases of polyglot aphasia have also suggested that the linguistic representation of L1 and L2 in polyglots might involve separate neurofunctional circuits, and/or be the result of a dysfunction of a putative switching mechanism between the languages. This assumption is based on evidence that, after insult, polyglot patients may selectively recover one language, while showing severe aphasic symptoms for the other language (Abutalebi et al., 2000; Aglioti, 1996; Aglioti \& Fabbro, 1993; Paradis, 1989). In general, although the role of the right hemisphere in bilinguals' language processing remains controversial, there is a great deal of evidence supporting a differential intrahemispheric representation for monolingual and bilingual speakers. For example, Dehaene and colleagues (Dehaene et al., 1997) used fMRI to assess intersubject variability in the cortical representation of language comprehension in moderately fluent French / English bilinguals while they listened to stories in the two languages. They found that while the first language (L1) activated a similar set of areas in the left temporal lobe in all subjects, the second language (L2) activated a highly variable network of the left and right temporal and frontal areas, with individual subjects varying from a standard left lateralization to a complete right hemispheric lateralization.

In summary, although further research is certainly needed to reach a definitive conclusion, it seems that linguistic functions are less lateralized in polyglots relative to monolinguals. The difference in lateralization in our study may be because of the interpreters' prolonged practice of simultaneous interpreting strategies. The difference may also be because multiple languages tend to be differentially represented in the brain, with a more extended right lateralization of linguistic functions in polyglots.

Considering that the orthographic task we used in the present experiments requires basic or simple linguistic processes, such as recognition of orthographic appearance and letter identification, and does not involve the sophisticated professional abilities of simultaneous interpreters, the data favour the hypothesis that the reduced left lateralization of linguistic functions in simultaneous interpreters may be attributed to the fact that they are polyglots, and not to the simultaneous interpretation task per se.

The possible limitations of this study refer to the presence of only female subjects in the interpreter group. It has been indeed demonstrated a lesser degree of lateralization for linguistic functions in the female than male brain. This effect might also possibly explain the prevalence of females in the simultaneous interpreter population, however further investigation is needed to reach a definitive conclusion on this matter.

In conclusion, the present data provide evidence of a lack of right hand preference for right-handed interpreters during an orthographic detection task. This finding might possibly reflect, for polyglots, a reduced hemispheric asymmetry for linguistic functions, normally lateralized to the left hemisphere, as clearly shown by the strong right lateral preference exhibited by the monolingual control group.

\section{Acknowledgements}

We are grateful to Giuliana Cosma Leoni and Alberto Zani for their kind support. Funded by MIUR grants.

\section{References}

Abutalebi, J., Miozzo, A., \& Cappa, S. F. (2000). Do subcortical structures control language selection in bilinguals Evidence from pathological language mixing. Neurocase, 6, 101-106.

Aglioti, S., Beltramello, A., Girardi, F., \& Fabbro, F. (1996). Neurolinguistic and follow-up study of an unusual pattern of recovery from bilingual subcortical aphasia. Brain, 119, 1551-1564. doi:10.1093/brain/119.5.1551

Aglioti, S., \& Fabbro, F. (1993). Paradoxical selective recovery in a bilingual aphasic following subcortical lesions. Neuroreport, 4, 1359-1362. doi:10.1097/00001756-199309150-00019

Chernigovskaya, T. V., Balonov, L. J., \& Deglin, V. L. (1983). Bilingualism and brain functional asymmetry. Brain and Language, 20, 195-216. doi:10.1016/0093-934X(83)90042-1

Cohen, L., \& Dehaene, S. (2004). Specialization within the ventral stream: The case for the visual word form area. NeuroImage, 22 , 466-476. doi:10.1016/j.neuroimage.2003.12.049

Cohen, L., Lehericy, S., Chochon, F., Lemer, C., Rivaud, S., \& Dehaene, S. (2002). Language-specific tuning of visual cortex. Functional properties of the Visual Word Form Area, 125, 1054-1069. doi:10.1097/00001756-199712010-00030

Dehaene, S., Dupoux, E., Mehler, J., Cohen, L., Paulesu, E., Perani, D., et al. (1997). Anatomical variability in the cortical representation of first and second language. NeuroReport, 8, 3809-3815. doi:10.1097/00001756-199712010-00030

Fabbro, F., Gran, B., \& Gran, L. (1991). Hemispheric specialization for semantic and syntactic components of language in simultaneous interpreters. Brain and Language, 41, 1-42. doi:10.1016/0093-934X(91)90108-D

Fabbro, F., Gran, L., Basso, G., \& Bava, A. (1990). Cerebral lateralization in simultaneous interpretation. Brain and Language, 39, 69-89. doi:10.1016/0093-934X(90)90005-2

Flowers, D. L., Jones, K., Noble, K., VanMeter, J., Zeffiro, T. A., Wood, F. B., et al. (2004). Attention to single letters activates left extrastriate cortex. NeuroImage, 21, 829-839. doi:10.1016/j.neuroimage.2003.10.002

Gran, L., \& Fabbro, F. (1989). Cerebral lateralization for syntactic and semantic components in L1 (Italian) and L2 (English) in interpreting students: Training implications for simultaneous interpretation. Paper presented at the Coming of age, Proceedings of the 30th Annual ATA Conference.

Green, A., Nicholson, N. S., Vaid, J., White, N., \& Steiner, R. (1990). Hemispheric involvement in shadowing vs. interpretation: A time-sharing study of simultaneous interpreters with matched bilingual and monolingual controls. Brain and Language, 39, 107-133. doi:10.1016/0093-934X(90)90007-4

Hugdahl, K., \& Davidson, R. J. (2003). Brain asymmetry II. MA, Cambridge: MIT Press.

Lambert, S. (1989). Simultaneous interpreters: One ear may be better than two. Canadian Association of Translation Studies, TTR, 2, 109 116.

Oldfield, R. C. (1971). The assessment and analysis of handedness: The Edinburgh inventory. Neuropsychologia, 9, 97-113. doi:10.1016/0028-3932(71)90067-4

Paradis, M. (1989). Bilingual and polyglot aphasia. In F. Boller \& J. Grafman (Eds.), Handbook of Neuropsychology, 2, 117-140.

Polk, T. A., Stallcup, M., Aguirre, G. K., Alsop, D. C., Esposito, M. D., Detre, J. A., et al. (2002). Neural specialization for letter recognition. Journal of Cognitive Neuroscience, 14, 145-159. doi:10.1162/089892902317236803

Proverbio, A. M., Čok, B., \& Zani, A. (2002). Electrophysiological measures of language processing in bilinguals. Journal of Cognitive Neuroscience, 14, 994-1017. doi:10.1162/089892902320474463

Proverbio, A. M., Leoni, G., \& Zani, A. (2004). Language switching mechanisms in simultaneous interpreters: An ERP study. Neuropsychologia, 42, 1636-1656.

doi:10.1016/j.neuropsychologia.2004.04.013

Proverbio, A. M., Adorni, R., \& Zani, A. (2007). The organization of 
multiple languages in polyglots: Interference or independence. Journal of Neurolinguistics, 20, 25-49.

doi:10.1016/j.jneuroling.2006.01.003

Proverbio, A. M., Wiedemann, F., Adorni, R., Rossi, V., Del Zotto, M., \& Zani, A. (2007). Dissociating object familiarity from linguistic properties in mirror word reading. Behavioral and Brain Functions, 3, 43. doi:10.1186/1744-9081-3-43

Proverbio, A. M., Zani, A., \& Adorni, R. (2008). The left fusiform area is affected by written frequency of words. Neuropsychologia, 46, 2292-2299. doi:10.1016/j.neuropsychologia.2008.03.024

Proverbio, A. M., Adorni, R., \& Zani, A. (2009). Inferring native language from early bio-electrical activity. Biological Psychology, 80,
52-63. doi:10.1016/j.biopsycho.2008.02.006

Rinne, J. O., Tommola, J., Laine, M., Krause, B. J., Schmidt, D., Kaasinen, V., et al. (2000). The translating brain: Cerebral activation patterns during simultaneous interpreting. Neuroscience Letters, 294, 85-88. doi:10.1016/S0304-3940(00)01540-8

Reicher, G. M. (1969). Perceptual recognition as a function of meaninfulness of stimulus material. Journal of Experimental Psychology, 81, 275-280. doi: $10.1037 / \mathrm{h} 0027768$

Xue, G., Chen, C., Jin, Z., \& Dong, Q. (2006). Cerebral asymmetry in the Fusiform areas predicted the efficiency of learning a new writing system. Journal of Cognitive Neuroscience, 18, 923-931. doi:10.1162/jocn.2006.18.6.923 